\title{
Patterns of diastolic dysfunction in left ventricular hypertrophy
}

\author{
LEONARD M SHAPIRO, * DEREK G GIBSON $\dagger$ \\ From the National Heart ${ }^{\star}$ and Brompton $\dagger$ Hospitals, London
}

SUMMARY The relative sensitivities of and interrelations between different measurements of diastolic function were studied in 50 patients with left ventricular hypertrophy diagnosed on anatomical grounds. Isovolumic relaxation time, the interval from minimum cavity dimension to mitral valve opening and relative dimension increase during this period, and the peak rate of dimension increase and wall thinning during rapid ventricular filling were measured by digitised $M_{\infty}^{\dot{\omega}}$ mode echocardiography. The relative heights of peak early diastolic and atrial velocities $(\mathrm{a} / \mathrm{E})$ and the time for decline of early diastolic velocity to half its peak value (velocity half time) were $\vec{T}$ measured on continuous wave and pulsed Doppler and the relative height of the "a" wave was $\frac{D}{0}$ measured by apexcardiogram. All sets of values except those of the interval from minimum dimension to mitral opening were unimodally distributed, and all differed significantly from those $\vec{\varnothing}$ in 20 age matched controls. The relative height of the "a" wave on the apexcardiogram ( $90 \%$ values ${ }^{\circ}$ were abnormal) was the most sensitive method of studying left ventricular diastolic function and peak rate of dimension increase was the least sensitive. Though none of the correlations was high, there were individual associations between peak rate of dimension increase, a/E, peak wall thinning rate, and velocity half time, and independently between delay in mitral valve opening and $\stackrel{\circ}{\mathbb{D}}$ dimension change during this period. Other values seemed to be independent of one another, suggesting a different physiological basis.

It is concluded that these various abnormal values do not reflect a single underlying disturbance of diastolic function. There are at least four possible discrete abnormalities: prolongation of $\frac{F}{0}$ isovolumic relaxation; incoordination during isovolumic relaxation; reduced rate of rapid filling; and an increase in the relative amplitude of the " $a$ " wave probably caused by increased passive stiffness. These may be present singly or in combination in any patient.

Left ventricular diastolic function may be abnormal in various cardiac conditions including coronary artery disease and hypertrophy.$^{1-3}$ These diastolic abnormalities may be associated with limitation of exercise tolerance, added heart sounds, and evidence of increased atrial contraction. They have been detected by an increasing number of invasive and non-invasive methods during isovolumic relaxation, rapid ventricular filling, diastasis, and left atrial systole. ${ }^{4-8}$ It is still not clear whether these all reflect, to a different extent, a single disturbance of diastole or whether there is more than one independent abnormality. We examined correlations between the

Requests for reprints to Dr Leonard $M$ Shapiro, National Heart Hospital, Westmoreland Street, London W1M 8BA.

Accepted for publication 9 November 1987 results of several of these investigations in an homogeneous group of patients with left ventricular 을 hypertrophy to determine the extent to which these $\rightarrow$ different measurements are interrelated. In this way we hoped to provide a framework in which this growing body of information might be assimilated, $\sigma$ and possibly to shed light on the nature of the underlying disturbances of diastole.

\section{Patients and methods}

STUDY POPULATION

An $M$ mode echocardiogram, apexcardiogram, and $\underset{T}{T}$ Doppler echocardiograms were recorded in $50 \stackrel{\circ}{\frac{0}{\mathbb{D}}}$ patients with left ventricular hypertrophy. We $\frac{\rho}{\Phi}$ selected patients in sinus rhythm in whom the left $\stackrel{\mathbb{Q}}{\varrho}$ ventricular mass exceeded the normal $95 \%$ 뭉 confidence interval. Patients were excluded if there $\delta$ 
was clinical evidence of coronary artery disease, mitral valve disease, valve regurgitation severe enough to increase cavity size, or evidence of regional abnormalities of wall motion on cross sectional echocardiography. The group with left ventricular hypertrophy was made up of 20 patients with hypertrophic cardiomyopathy, 24 with valvar or subvalvar aortic stenosis, and six with systemic hypertension. There were 27 male patients and 23 female patients, aged 12 to 74 years (mean 53 ).

Twenty subjects (11 male and nine female, aged 14-71 years) without heart disease underwent a similar cardiac investigation and served as normal controls.

\section{MODE ECHOCARDIOGRAM}

The patients were studied in the partial left lateral position. The examination was completed in 10 to 15 minutes, during which time there was no obvious change in haemodynamic state. Echocardiograms were recorded with simultaneous phonocardiograms and electrocardiograms at a paper speed of $10 \mathrm{~cm} / \mathrm{s}$. A left ventricular echocardiogram at the level of the tips of the mitral valve (to define onset of cusp opening) showing clear continuous echoes from both the septum and posterior wall was used for further analysis (fig 1). The thickness of the septum and posterior wall was measured $(\mathrm{cm})$ at end diastole $(Q$ wave) by the leading edge method. The timing of aortic valve closure was taken as the onset of the first high frequency vibration of the aortic second heart sound and isovolumic relaxation as the interval between this and the initial separation of the mitral valve leaflets. The interval from minimum cavity dimension to mitral valve opening was measured (ms) and the increase in transverse cavity dimension during that period was expressed as a percentage of the total dimension change during the cardiac cycle.

Echocardiograms were digitised ${ }^{6}$ by a Summagraphics digitiser and a Prime computer system. We

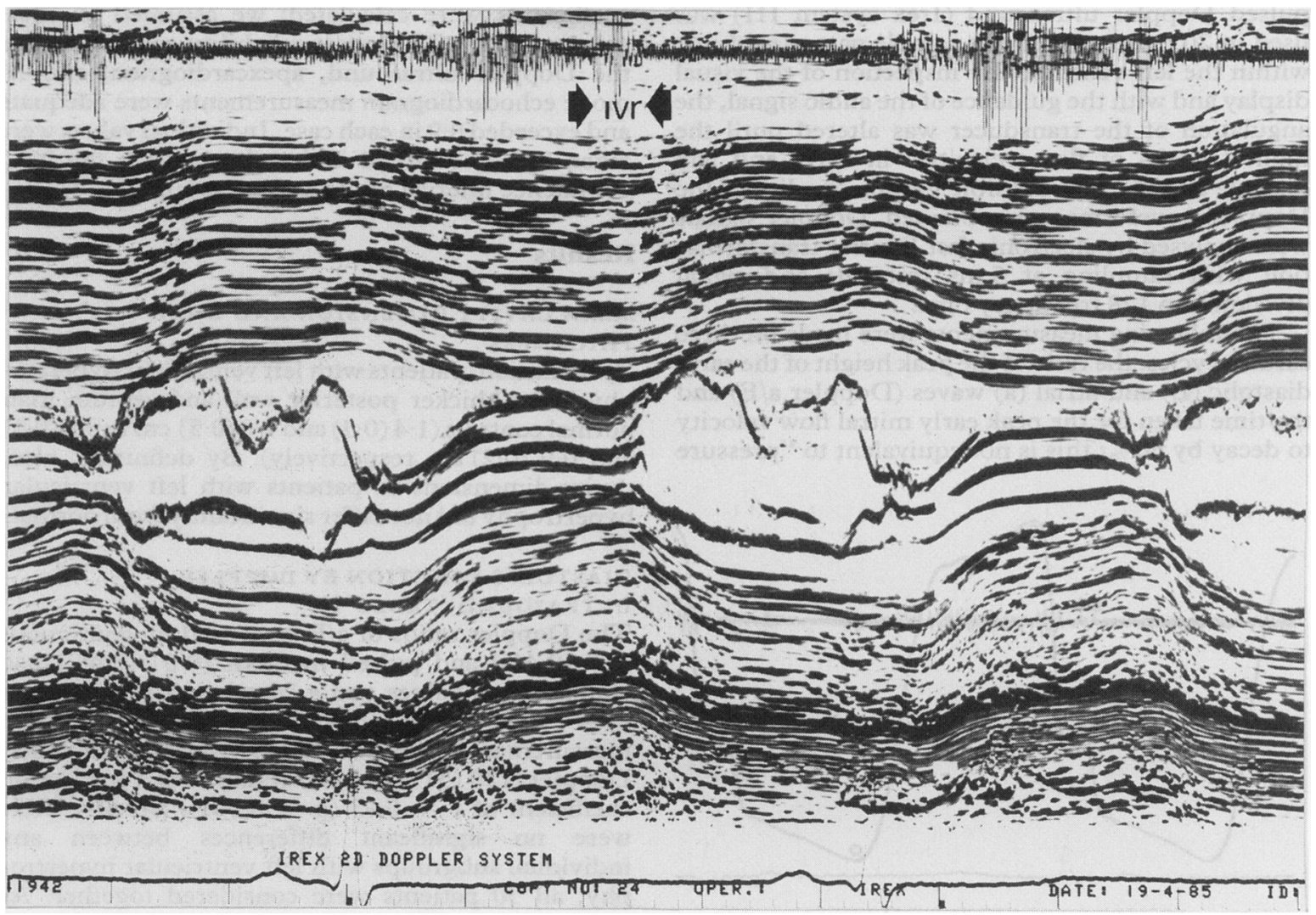

Fig $1 M$ mode echocardiogram in a patient with severe left ventricular hypertrophy caused by hypertrophic cardiomyopathy. Isovolumic relaxation time (ivr) is prolonged ( $95 \mathrm{~ms}$ ) but there is only minor delay in mitral valve opening relative to minimum cavity dimension without appreciable outward wall motion. Both the peak rate of dimension increase and posterior wall thinning are reduced. 
measured the peak rates $(\mathrm{cm} / \mathrm{s})$ of increase in dimension and posterior wall thinning during early diastole from the records.

\section{APEXCARDIOGRAM}

The apexcardiogram was recorded from the point of maximum cardiac impulse with the patient in the partial left lateral position. A transducer funnel that enclosed the impulse was selected $(2.5$ or $3 \mathrm{~cm})$. The system had a time constant of $4 \mathrm{~s}$ with a lower frequency limit of $0.05 \mathrm{~Hz}$. We calculated the ratio of the height of the A wave to the total deflection EO, (A/EO) from a mean of three cardiac cycles (fig 2).

\section{DOPPLER ULTRASOUND}

After a cross sectional echocardiographic study a continuous wave dual crystal Doppler transducer was placed at the point of maximum cardiac displacement. A $2 \mathrm{MHz}$ transducer with either Doptek or Vingmed equipment interfaced with Irex spectral analysis was used. In addition, duplex scanning with pulsed Doppler ultrasound (Irex system III) was used in 20 patients with the sample gate placed just within the left ventricle. By inspection of the visual display and with the guidance of the audio signal, the angulation of the transducer was altered until the highest peaks of flow velocities in early and late diastole and the best possible graphic quality of the Doppler waveforms were obtained. Doppler signals were analysed by electronic fast Fourier transformation with sampling at $5 \mathrm{~ms}$ intervals and signal division into 128 frequency bins.

The following measurements were made on three cardiac cycles: the ratio of the peak height of the early diastolic (E) and atrial (a) waves (Doppler a/E) and the time taken for the peak early mitral flow velocity to decay by $50 \%$; this is not equivalent to "pressure

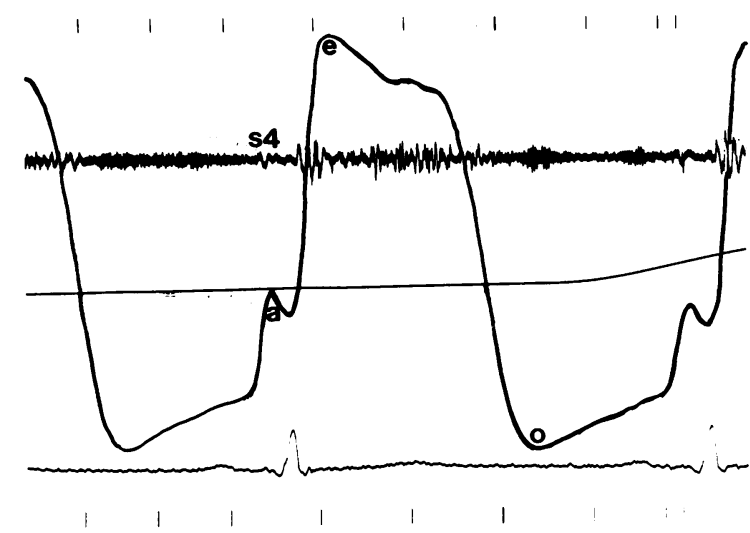

Fig 2 Apexcardiogram in the same patient as fig 1 showing a markedly accentuated " $a$ " wave coincidental with the phonocardiographic fourth heart sound (s4). half time" because in contrast with the stenotic atrioventricular valve the decline in flow velocity across a normal valve is not exponential and during the phase of deceleration the pressure gradient is likely to be reversed. The measurement has therefore been referred to as a velocity half time.

\section{STATISTICAL METHODS}

Values are quoted as mean (1 SD). Student's $t$ test is (unpaired) was used to test differences between normally distributed variables and the MannWhitney $U$ test between variables with a skewed distribution. The relation between continuously distributed variables was measured by Pearson's correlation coefficient $(r)$. Fisher's exact probability test was used to test the power of association and the Kolmogorov-Smirnov test to determine the nature of $\vec{\omega}$ numerical distribution. Measurement reliability was tested by the random selection of 10 sets of records and repetition of the measurements without prior knowledge of the previous results. Retest reliability coefficients were calculated; we assumed an ideal value of unity. The retest reliability coefficients for the Doppler ultrasound, apexcardiogram, and $M$ mode echocardiogram measurements were adequate and exceeded 0.9 in each case. Individual values were taken to be abnormal if they lay outside the $99 \%$ confidence interval of the normal group.

\section{Results}

MEAN CAVITY DIMENSION AND WALL

THICKNESS

By definition, patients with left ventricular hypertrophy had a thicker posterior wall and septum than normal controls $(1.4(0.3)$ and $1.8(0.5) \mathrm{cm}$ vs $0.8(0.1)$ and $0.9(0.2) \mathrm{cm}$ respectively). By definition, also, cavity dimensions in patients with left ventricular hypertrophy did not differ significantly from normal.

DIASTOLIC FUNCTION BY DOPP.LER

ULTRASOUND (TABLE 1)

The Doppler ratios of a/E measured by continuous $(0.9(0.46))$ and pulsed methods $(0.87(0.45))$ had 0 similar mean values and a correlation coefficient of $\tilde{N}$ $r=0.94(p<0.001)$. Velocity half times $\underset{\omega}{N}$ measurement by continuous $(119(50) \mathrm{ms})$ and pulsed $\sigma$ Doppler $(120(51) \mathrm{ms})$ were similar with a correlation 0 coefficient of $r=0.93(p<0.001)$. Because there $\overbrace{\overparen{D}}$ were no significant differences between any $\stackrel{\infty}{\cdot}$ individual subgroups with left ventricular hypertro- $\frac{T}{0}$ phy, all 50 patients were considered together. As expected they differed significantly from the normal $\frac{\rho}{\$}$ group: the Doppler ratio a/E was greater $(p<0.001) \stackrel{\varnothing}{\unrhd}$ with $15(30 \%)$ outside the normal $99 \%$ confidence interval and the velocity half time was reduced $\varnothing$ 


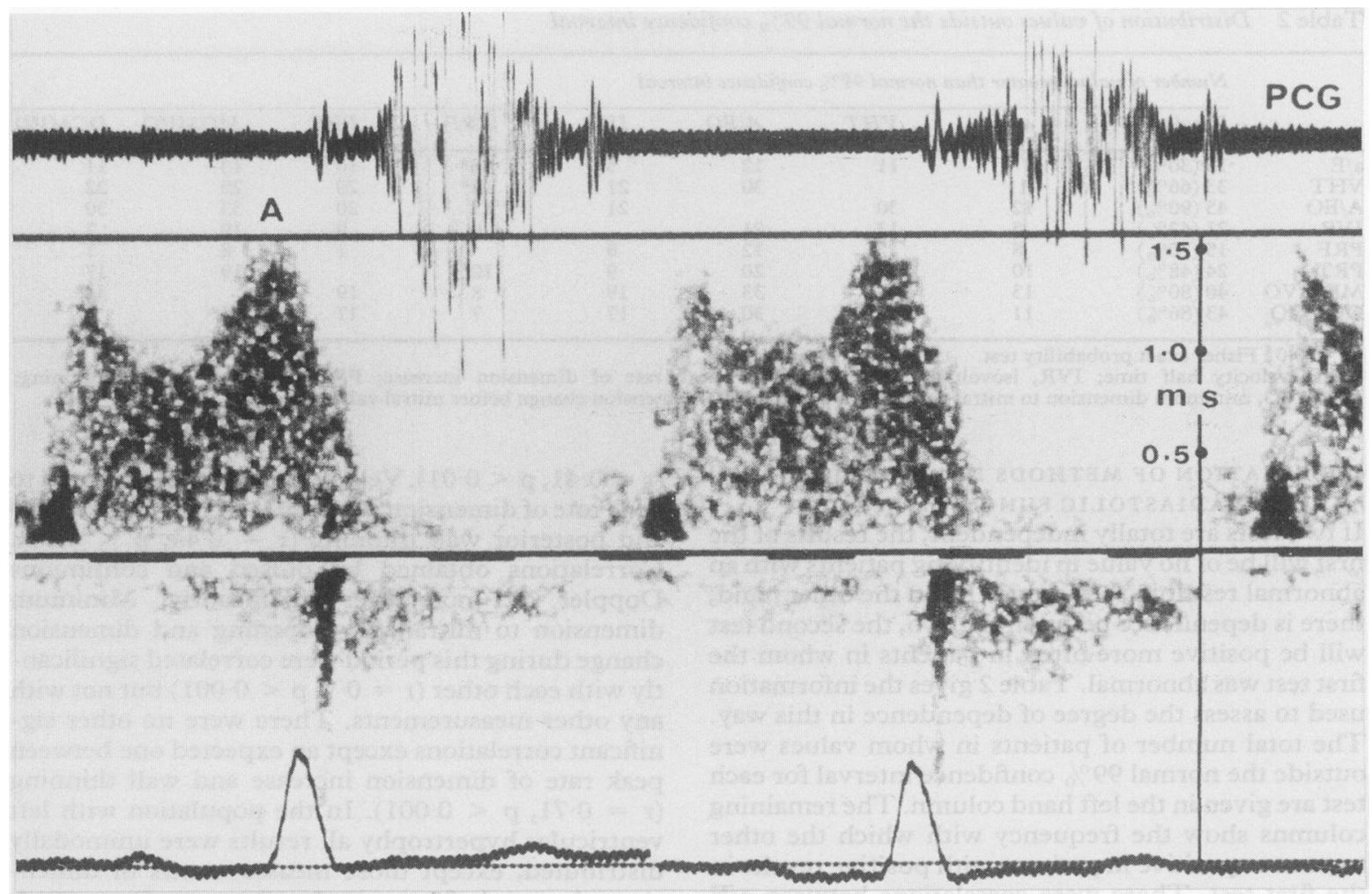

Fig 3 Apical continuous wave Doppler study showing a reduced velocity half time and a dominant $A$ wave in a patient with aortic stenosis.

$(p<0.001)$ with only 17 patients within the normal range (table 1).

\section{DIASTOLIC FUNCTION BY DIGITISED M MODE ECHOCARDIOGRAPHY}

Mean isovolumic relaxation time was longer in patients with left ventricular hypertrophy than in normal controls. Twenty one $(42 \%)$ patients had values above the upper $99 \%$ confidence interval for normal and six had values below the lower limit. In patients with left ventricular hypertrophy mitral valve opening was delayed by 77 (45) ms relative to minimum dimension (normal 10 (3), p $<0.001$ ), with two having values below and 40 values above the normal $99 \%$ confidence interval. In patients with left ventricular hypertrophy the change in dimension before mitral valve opening was $20(19) \%$ compared with the normal of $0(3) \%$, with one patient having values below and 32 values above the normal $99 \%$ confidence interval. The mean values for the peak rate of dimension increase and wall thinning were both less than normal ( $<<0.001$ ) with 15 and 24 patients respectively having values below the normal range.
DIASTOLIC FUNCTION BY APEXCARDIOGRAPHY Only five patients with left ventricular hypertrophy had a normal A/EO and in this group the mean value was greater than normal $(p<0.001)$.

Table 1 Comparison of diastolic function in normal controls and patients with left ventricular hypertrophy

\begin{tabular}{|c|c|c|}
\hline & Normal & $\begin{array}{l}\text { Left ventricular } \\
\text { hypertrophy }\end{array}$ \\
\hline $\begin{array}{l}\text { No } \\
\text { a/E }(\%) \\
\text { Velocity half time (ms) } \\
\text { A/EO (\%) } \\
\text { Isovolumic relaxation time (ms) } \\
\text { Minimum dimension (ms) to } \\
\text { MVO }\end{array}$ & $\begin{array}{ll}20 & \\
0.52 & (0 \cdot 12) \\
62 & (14) \\
0.07 & (0 \cdot 02) \\
57 & (8) \\
10 & (3)\end{array}$ & $\begin{array}{cl}50 & \\
0 \cdot 71 & (0 \cdot 4) \\
126 & (38) \\
0 \cdot 26 & (0 \cdot 02) \\
90 & (38) \\
77 & (45)\end{array}$ \\
\hline $\begin{array}{l}\text { Dimension change before } \\
\text { MVO }(\%)\end{array}$ & (3) & (19) \\
\hline $\begin{array}{l}\text { Peak rate }(\mathrm{cm} / \mathrm{s}) \text { of dimension } \\
\text { increase }\end{array}$ & (3) & $(3 \cdot 6)$ \\
\hline Peak rate $(\mathrm{cm} / \mathrm{s})$ of wall thinning & (2) & $(2 \cdot 4)$ \\
\hline
\end{tabular}

a/E, Doppler ratio of "a" to E waves; MVO, mitral valve opening; A/EO, apexcardiographic relative height of "a" wave. Values are mean (1 SD). 
Table 2 Distribution of values outside the normal $99 \%$ confidence interval

\begin{tabular}{|c|c|c|c|c|c|c|c|c|c|}
\hline & \multicolumn{9}{|c|}{ Number of values greater than normal $99 \%$ confidence interval } \\
\hline & Total & $a / E$ & $V H T$ & $A / E O$ & $I V R$ & $P R F$ & $P R T$ & $M D M V O$ & $\overline{D C M V O}$ \\
\hline $\begin{array}{l}\text { a/E } \\
\text { VHT } \\
\text { A/EO } \\
\text { IVR } \\
\text { PRF } \\
\text { PRT } \\
\text { MDMVO } \\
\text { DCMVO }\end{array}$ & $\begin{array}{l}15(30 \%) \\
33(66 \%) \\
45(90 \%) \\
21(42 \%) \\
15(30 \%) \\
24(48 \%) \\
40(80 \%) \\
43(86 \%)\end{array}$ & $\begin{array}{r}11 \\
12 \\
5 \\
8 \\
10 \\
13 \\
11\end{array}$ & $\begin{array}{l}11 \\
30 \\
13 \\
12 \\
17 \\
25 \\
22\end{array}$ & $\begin{array}{l}12 \\
30 \\
21 \\
12 \\
20 \\
33 \\
30\end{array}$ & $\begin{array}{r}5 \\
21 \\
21 \\
6 \\
9 \\
19 \\
17\end{array}$ & $\begin{array}{c}8^{\star} \\
13^{\star} \\
13 \\
6 \\
10^{\star} \\
8 \\
7\end{array}$ & $\begin{array}{r}10 \\
20 \\
20 \\
9 \\
1 \\
19 \\
17\end{array}$ & $\begin{array}{r}13 \\
25 \\
33 \\
19 \\
8 \\
19 \\
34^{\star}\end{array}$ & $\begin{array}{c}11 \\
22 \\
30 \\
7 \\
7 \\
17 \\
34^{\star}\end{array}$ \\
\hline
\end{tabular}

${ }^{\star} \mathrm{p}<0.01$ Fisher exact probability test.

VHT, velocity half time; IVR, isovolumic relaxation; PRF, peak rate of dimension increase; PRT, peak rate of wall thinning; MDMVO, minimum dimension to mitral valve opening; DCMVO, dimension change before mitral valve opening.

\section{CORRELATION OF METHODS IN DETECTING}

ABNORMAL DIASTOLIC FUNCTION

If two tests are totally independent, the results of the first will be of no value in identifying patients with an abnormal result in the second. If, on the other hand, there is dependence between the two, the second test will be positive more often in patients in whom the first test was abnormal. Table 2 gives the information used to assess the degree of dependence in this way. The total number of patients in whom values were outside the normal $99 \%$ confidence interval for each test are given in the left hand column. The remaining columns show the frequency with which the other tests were positive in patients with positive results in the first test. There were correlations between $a / E$ and peak rate of dimension increase, between velocity half time and peak rate of dimension increase, and between peak rate of dimension increase and peak thinning rate. A separate relation was noted between delay in mitral valve opening with respect to minimum dimension and the dimension change during that period (all $p<0.01$ by Fisher's exact probability test). For the remaining tests positive results in the second test were unrelated to positive results in the first test.

When the results in individual patients were examined the conclusion was similar. The Doppler a/E ratio correlated with velocity half time $(r=0.50$, $p<0.001)$ and with peak rate of dimension increase $(\mathrm{r}=0.41, \mathrm{p}<0.01)$. Velocity half time was related to peak rate of dimension increase $(r=0.51, p<0.001) \stackrel{\infty}{\infty}$ and posterior wall thinning $(\mathrm{r}=0.44, \mathrm{p}<0.01)$. 우 Correlations obtained by pulsed and continuous Doppler did not differ significantly. Minimum $\rightarrow$ dimension to mitral valve opening and dimension change during this period were correlated significantly with each other $(r=0.7, p<0.001)$ but not with any other measurements. There were no other significant correlations except an expected one between peak rate of dimension increase and wall thinning $(\mathrm{r}=0.71, \mathrm{p}<0.001)$. In the population with left ventricular hypertrophy all results were unimodally distributed, except those measurements of dimension change before mitral valve opening, which showed peaks at $15 \%$ and $50 \%$ total dimension change respectively. The former is within the normal $99 \%$ confidence interval and the latter outside it. There was no predominance of any patient group in either peak, however.

\section{Discussion}

Diastolic left ventricular function is often abnormal in patients with left ventricular hypertrophy, ${ }^{39}$ and more abnormalities are reported as new methods are described for its study. It is not clear, however, how all these measurements, often referred to as "parameters" of diastolic function, relate to one

Table 3 Correlation between measurements of diastolic function in patients with left ventricular hypertrophy

\begin{tabular}{|c|c|c|c|c|c|c|c|c|}
\hline & $a / E(C W)$ & $V H T(C W)$ & $A / E O$ & $I V R$ & $P R F$ & $P R T$ & $M D M V O$ & $D C M V O$ \\
\hline $\begin{array}{l}\text { VHT (CW) } \\
\text { a/E (CW) } \\
\text { VHT (PW) } \\
\text { a/E (PW) } \\
\text { A/EO }\end{array}$ & $\begin{array}{l}0.5^{\star} \\
0.87 \ddagger \\
0.94 \ddagger \\
0.37 \dagger\end{array}$ & $\begin{array}{l}0.5^{\star} \\
0.94 \ddagger \\
0.83 \ddagger \\
0.16\end{array}$ & $\begin{array}{l}0 \cdot 16 \\
0.37 \dagger \\
0 \cdot 27 \\
0.34\end{array}$ & $\begin{array}{l}0.02 \\
0.17 \\
0.105 \\
0.21 \\
0.05\end{array}$ & $\begin{array}{l}0.5^{\star} \\
0.4^{\star} \\
0.58^{\star} \\
0.55^{\star} \\
0.01\end{array}$ & $\begin{array}{l}0.44^{\star} \\
0.13 \\
0.51 \star \\
0.3 \\
0.01\end{array}$ & $\begin{array}{l}0.03 \\
0.04 \\
0.2 \\
0.01 \\
0.03\end{array}$ & $\begin{array}{l}0.01 \\
0.08 \\
0.2 \\
0.1 \\
0.1\end{array}$ \\
\hline
\end{tabular}

CW and PW represent continuous and pulsed wave Doppler recordings.

Significance of $r$ value: ${ }^{\star} p<0.01 ; \neq p<0.05 ; \ddagger p<0.001$.

See footnote to table 2 for abbreviations. 
another. One view is that they all reflect, to a greater or lesser extent, some single underlying disturbance, such as "impaired relaxation"10 or "reduced compliance"." If so, the various methods can only diverge in individual patients because of incidental factors such as their relative indirectness or simply random error. An alternative view is that two or more discrete disturbances of diastolic function exist, whose extent can vary independently between patients. We aimed to study this problem by making a series of measurements of diastolic left ventricular function in a homogeneous group of patients with left ventricular hypertrophy confirmed by well defined anatomical criteria. The majority had outflow tract obstruction or hypertrophic cardiomyopathy. The methods were designed to cover all aspects of diastole from isovolumic relaxation to atrial systole, and we were interested not only in their relative sensitivity in detecting any abnormality, but also in their distribution and the extent to which their results were independent of one another.

Our results confirm that all the methods we used frequently gave values outside the $99 \%$ confidence interval of an age matched normal population. We chose $99 \%$ rather than $95 \%$ intervals because our normal population was not large enough to allow us to derive the relation between these normal intervals and age, and because we used them to identify abnormal values in individual patients. ${ }^{1213}$ Some methods gave abnormal values much more often than others. Those that were most often abnormal were an increase in the relative height of the "a" wave on the apexcardiogram and delay in mitral valve opening with respect to minimum cavity dimension. The relative height of the " $a$ " wave on the Doppler echocardiogram (a/E) and peak rate of dimension increase on the $M$ mode echocardiogram were abnormal least often. Intermediate were the duration of isovolumic relaxation, the rate of velocity decline at the end of the rapid phase of left ventricular filling, and peak rate of thinning of the posterior left ventricular wall.

Although values for these different measurements did not correlate closely in individual patients, there were significant associations. Thus a reduced rate of dimension increase was much commoner in patients with a prolonged flow velocity decline than in those in whom it was normal; $\mathrm{a} / \mathrm{E}$ ratio and peak rate of dimension increase were similarly associated. This suggests that these three quantities were not independent of one another. In the same way, the delay in mitral valve opening with respect to minimum dimension was independently related to the extent of left ventricular dimension increase during the same period, but not with isovolumic relaxation time itself. Indeed, we could not demonstrate any association between isovolumic relaxation time or the relative height of the "a" wave on the apexcardiogram and any other of the variables that we investigated. The measurements themselves were all internally consistent, and values often departed considerably from normal in patients with left ventricular hypertrophy. We are not therefore inclined to ascribe this independence simply to extensive noise or physiological variation confounding our attempts to determine the extent of a single disturbance, but rather we consider it provides evidence that there is more than one discrete determinant of diastolic function.

Most of the measurements we made were unimodally distributed throughout the patient population. Those of dimension increase before mitral valve opening were exceptional in falling into two groups, one with values only slightly higher than normal and the other in which they were considerably more disturbed. An increase in cavity dimension during isovolumic relaxation must represent a change in ventricular cavity shape and not the effect of filling, so both it, and also the delay in mitral valve opening with which it is associated, are likely to be the result of incoordination during early diastole. Although its mechanism is not clear, it appears that partial forms of this aspect of incoordination do not exist, and that it is either present in fully developed form or absent altogether. By contrast, prolongation of isovolumic relaxation time not only behaved independently of these other two measures but also was continuously distributed, and so is likely to have some other mechanism. This distinction has practical importance, since the interval from minimum dimension to mitral valve opening has been used as a measure of isovolumic relaxation time, and has even been termed "isovolumic relaxation time index". ${ }^{14}$ Our results not only demonstrate that the two measurements behave independently in patients with left ventricular hypertrophy in whom this index has been used, but suggest that they may even have different underlying mechanisms.

The basis of the apexcardiogram during diastole is known to depend mainly on left ventricular pressure. ${ }^{15}$ An increase in the relative height of the " $a$ " wave is thus likely to reflect the combined effect of a larger atrial stroke volume and a stiffer left ventricular cavity. It was thus interesting that this relatively direct measurement proved so much more sensitive than the Doppler determination of relative blood velocity at the same time. This discrepancy emphasizes how little is known of what determines atrial flow rate. It is more a manifestation of atrial systolic rather than ventricular diastolic function. Flow velocity presumably increases only when some abnormal ventricular diastolic property acts as an 
increase in atrial afterload, though how a change in atrial loading conditions affects its systolic function is not well understood. Our results make it unlikely that this intermediate factor is simply an increase in ventricular end diastolic pressure. Of the measurements with which it was interrelated, twoflow velocity decline and peak rate of dimension increase-were both measures of rapid filling, and on this basis it may be that the a/E ratio depends more on a low early diastolic flow velocity, with the increase during atrial systole being merely compensatory.

Ventricular loading conditions greatly affect measurements of diastolic function, particularly relaxation. ${ }^{16}$ The question thus arises as to whether this load dependence could cause the discrepancies between measurements in individual patients. Though load dependence may modify the rate of relaxation, if relaxation rate itself is the only determinant of diastolic function, variation in loading could not explain discrepancies between methods unless it was actually altering at the time that measurements were made. Changes in loading can also alter individual and potentially independent measurements by a variety of other mechanisms. Isovolumic relaxation time shortens when left atrial pressure rises, though it is little affected by aortic pressure. ${ }^{17}$ Ventricular filling rate increases when atrioventricular pressure gradient rises, ${ }^{10}$ and an increase in left atrial pressure increases the prominence of the " $a$ " wave on the apexcardiogram. ${ }^{15} \mathrm{Had}$ loading conditions been a significant source of variance, therefore, correlation would have been expected between these three variables. Its conspicuous absence suggests that other factors must be involved.

Several conclusions can be drawn from these results about diastolic abnormalities of the left ventricle occurring in uncomplicated hypertrophy. In having two subgroups, incoordination differs fundamentally from the remainder of the variables which were continuously distributed. The overall sensitivity of the different measurements in detecting abnormalities varied widely, ranging from less than 50 to more than $90 \%$. Finally, individual measurements appeared to associate themselves into four sets giving results that were independent of one another. Even at the present stage, therefore, our findings are incompatible with the original premise that all diastolic abnormalities reflect, to some degree, the severity of a single, scalar disturbance of left ventricular function, whatever its nature. Rather, they suggest the existence of a series of independent disturbances, present to varying degrees in individual patients. We suggest that they might be classified as follows: type 1 presenting as prolongation of isovolumic relaxation time, which, in the absence of abnormal loading conditions, is likely to be due to a reduction in the rate of ventricular pressure fall. Incoordinate wall motion or stress reduction during isovolumic relaxation is responsible for type 2 abnormalities, while type 3 disturbances reflect a reduced rate of rapid filling. Type 4 , here represented by a relative increase in the height of the "a" wave on the apexcardiogram, is probably caused by increased passive stiffness of the ventricular cavity. ${ }^{18}$ Other abnormalities certainly exist within each group, and this classification allows for further independent mechanisms to be described. Future work should therefore be directed towards defining other abnormalities in greater detail and understanding when and why they occur.

\section{References}

1 Gibson DG, Prewitt TA, Brown DJ. Analysis of left ventricular wall movement during isovolumic relaxation and its relation to coronary artery disease. $\mathrm{Br}$ Heart $J$ 1976;38:1010-9.

2 Sanderson JE, Gibson DG, Brown DJ, Goodwin JF. Left ventricular filling in hypertrophic cardiomyopathy. An angiographic study. $\mathrm{Br}$ Heart $\mathrm{J}$ 1977;39:661-70.

3 Gibson DG, Traill TA, Hall RJC, Brown DJ. Echocardiographic features of secondary left ventricular hypertrophy. Br Heart J 1979;41:54-9.

4 Hammermeister KE, Warbasse JR. The rate of change of left ventricular volume in man. II Diastolic events in health and disease. Circulation 1974;49:739-47.

5 Bonow RO, Frederick TM, Bacharach SL, et al. Atrial systole and left ventricular filling in hypertrophic cardiomyopathy. Am J Cardiol 1983;51:1386-91.

6 Gibson DG, Brown D. Measurement of instantaneous left ventricular dimension and filling rate in man, using echocardiography. Br Heart J 1973;35:1141-9.

7 Hatle L, Angelsen B. Doppler ultrasound in cardiology. Philadelphia: Lea and Febiger, 1982.

8 Spirito P, Maron B, Bonow RO. Noninvasive assessment of left ventricular diastolic function: comparative analysis of Doppler echocardiographic and radionuclide angiographic techniques. $\mathrm{J} \mathrm{Am} \mathrm{Coll}$ Cardiol 1986;7:518-26.

9 Shapiro LM, Moore RB, Logan-Sinclair RB, Gibson DG. Relation of regional echo amplitude to left ventricular function and the electrocardiogram in left ventricular hypertrophy. Br Heart J 1984;52:99-105.

10 Ishida Y, Meisner JS, Tsujioka K, et al. Left ventricular filling dynamics: influence of left ventricular relaxation and left atrial pressure. Circulation 1986;74: 187-96.

11 Danford DA, Huhta JC, Murphy DJ. Doppler echocardiographic approaches to ventricular diastolic function. Echocardiogr 1986;3:33-40.

12 Miyatake K, Okamoto M, Kinoshita N, et al. Augmentation of atrial contribution to left ventricular inflow with ageing as assessed by intracardiac Doppler flowmetry. Am J Cardiol 1984;53:586-9.

13 Henry WL, Gardin JM, Ware JH. Echocardiographic 
measurements in normal subjects from infancy to old age. Circulation 1980;62:1054-60.

14 Hanrath P, Mathey DG, Siefert R, Bleifeld W. Left ventricular relaxation and filling pattern in different forms of left ventricular hypertrophy: an echocardiographic study. Am J Cardiol 1980;45:15-23.

15 Denef B, Popeye R, de Geest H, Kesteloot H. On the clinical value of calibrated apexcardiography. Circulation 1975;51:541-51.

16 Brutsaert DL, Housmans PR, Goethals MA. Dual control of relaxation. Its role in the ventricular function in the mammalian heart. Circ Res 1980;47:637-52.

17 Mattheos M, Shapiro E, Oldershaw PJ, Sacchetti R, Gibson DG. Non-invasive assessment of changes in left ventricular relaxation by combined phono-, echo-, and mechanocardiography. $\mathrm{Br}$ Heart $J$ 1982;47:253-60.

18 Grossman W, Stefadouros MA, McLaurin LP, Rolett EL, Young DT. Quantitative assessment of left ventricular stiffness in man. Circulation 1973;37: 567-74. 